\title{
The efficacy of methylprednisolone treatment in inflammatory response during cardiopulmonary bypass: a systematic review
}

\begin{abstract}
Background: Due to the conflicting results about beneficial effects of methylprednisolone administration on inflammatory response following cardiopulmonary bypass (CPB), this research is aim at evaluate the efficacy of methylprednisolone treatment in inflammatory response during $\mathrm{CPB}$.
\end{abstract}

Methods: Databases, including PubMed, EMBASE, Cochrane Central and Web of Science, were searched for relevant studies up to April 1, 2016 without language restriction. Randomized controlled trials about the efficacy of methylprednisolone treatment in inflammatory response during $\mathrm{CPB}$ were included in this systematic review.

Results: The descriptive analysis showed that methylprednisolone can reduce TNF- $\alpha$, IL-6, and IL-8 release.

Conclusion: Methylprednisolone is beneficial to reduce the inflammatory response during the CPB. Randomized controlled trials which have high methodological quality are needed to confirm this conclusion.

Keywords: methylprednisolone, inflammatory response, cardiopulmonary bypass, systematic review, randomized controlled trials
Volume 3 Issue 5 - 2017

\author{
Hong Yan Sun, Lian Liu, Fan Li, Siping Yu, Qiu \\ Xia Liu, Xiao Ling Yang \\ Nursing School, Southwest Medical University, China
}

Correspondence: Hong Yan Sun, Nursing School, Southwest Medical University, Luzhou, Si chuan, No. 3, 319 section of Zhong Shan Road, Jiangyang District, Luzhou 646000, China, Email sunhongyan234@I63.com

Received: September 09, 2017 | Published: October 03, 2017

\section{Introduction}

Cardiopulmonary bypass (CPB) a complicated pathophysiology environment, exposures to nonphysiologic surfaces in the pump circuit, hemolysis, and ischemia-reperfusion injury combine to originate a complicated cascade which includes proinflammatory cytokines, anti-inflammatory cytokines, and products of neutrophil activation. ${ }^{1}$ The systemic inflammatory response to CPB is launched by touching blood with the extracorporeal circuit, ischemia-reperfusion injury, surgical trauma, and release of endotoxin. ${ }^{2}$ The cytokines, including interleukin-6 (IL-6), interleukin-8 (IL-8), and tumor necrosis factor- $\alpha$ $(\mathrm{TNF}-\alpha)$, are involved in the induction of the inflammatory response in patients undergoing cardiac surgery. ${ }^{3}$ Corticosteroids are generally used in patients undergoing $\mathrm{CPB}$ attenuate systemic inflammatory response. However, a large retrospective observational analysis showed that corticosteroids may aggrandize morbidity; particularly in lower-risk pediatric cardiac surgery patients. ${ }^{4}$ Beneficial effects of methylprednisolone administration on inflammatory response following CPB always have produced conflicting results. Minority of evidence hitherto could state clearly the inflammatory response to methylprednisolone treatment in patients undergoing CPB. Therefore, we did a systematic review to evaluate efficacy of methylprednisolone treatment in inflammatory response during $\mathrm{CPB}$.

\section{Methods}

\section{Search strategy and selection criteria}

Four electronic databases were searched for articles published up to April 1, 2016 without language restriction, containing Pub Med, EMBASE, Cochrane Central and Web of Science. The search terms used are presented as follows: ['heart-lung bypass' OR 'bypass, heart-lung' OR 'bypasses, heart-lung' OR 'heart lung bypass' OR 'heart-lung bypasses' OR 'bypass, cardiopulmonary' OR 'bypasses, cardiopulmonary' OR 'cardiopulmonary bypasses'] AND ['metipred' OR ‘6-methylprednisolone' OR '6 methylprednisolone' OR 'urbason' OR 'medrol'] AND ['random' OR 'randomized controlled trial']. The reference lists of relevant reviews were also hand searched to identify additional studies. We included studies according to the following inclusion criteria:

A. Participants undergoing cardiopulmonary bypass;

B. Patients were randomized grouped into the methylprednisolone group (without other drug) and the placebo group (with or without placebo );

C. Not less than one outcome ((tumor necrosis factor- $\alpha($ TNF- $\alpha$ ), interleukin-1 $\beta$ (IL-1 $\beta$ ), interleukin-6 (IL-6), interleukin-8 (IL8), interleukin-10 (IL-10));

D. Randomized controlled trials. The articles which were retrospective studies, observational studies, case series, reviews, comments, duplicate published articles, and studies without original data were also eliminated.

\section{Data extraction}

Two authors independently collected on the following data: the first author, publication year, sample size, study design, patient characteristics, the methylprednisolone group (dosage, duration), the placebo group (dosage, duration), and measured outcome (tumor necrosis factor- $\alpha$ (TNF- $\alpha$ ), interleukin-1 $\beta$ (IL-1 $\beta$ ), interleukin-6 (IL6), interleukin-8 (IL-8), interleukin-10 (IL-10)). We made a detailed discussion when disagreements of this process occurred. 


\section{Quality assessment}

The risk of bias was assessed by two authors independently using the Cochrane risk of bias tools, containing six items: selection bias, performance bias, detection bias, attrition bias, reporting bias, other bias $^{5}$ In addition, we adopted the Jadad scale to assess quality of included studies. The Jadad scale which is constantly used to assess the quality of randomized controlled trials is a 5-point scale, including three items: the generation of random grouping method, double blind, and reasons for withdrawals and dropouts. Five points is the maximum score; the studies with $0-2$ points belong to low quality, and the studies with 3-5 points belong to high quality.

\section{Data synthesis}

We did not do a meta-analysis because of greater heterogeneity and without suitable data. Hence, we adopted a descriptive analysis instead of meta-analysis.

\section{Results}

The initial search identified 406 records, but this systematic review only included 6 trials ${ }^{6-11}$ by the inclusion criteria and exclusion criteria as shown in Figure 1. Patient characteristics and Jadad scale were summarized in Table 1 the risk of bias as noted in Table 2. The quality of the studies was various; the majority was poor quality studies, and there were only 2 high quality trials. ${ }^{10,11}$ Two trials 7,8 reported levels of TNF- $\alpha$. Ayda et al. ${ }^{7}$ showed that levels of TNF- $\alpha$ $(p<0.05)$ at 24 hours after CPB were significantly greater in the placebo group than in the methylprednisolone group. In addition, Jale Bengi et al. ${ }^{8}$ showed that levels of TNF- $\alpha(\mathrm{p}<0.05)$ at 24 hours after CPB were significantly greater in the placebo group than in the methylprednisolone group. Only one trial 7 reported levels of IL-1 $\beta$ at 24 hours after $\mathrm{CPB}$, but there was no statistically significant difference between the methylprednisolone group and the placebo group. Six studies $^{6-11}$ reported levels of IL-6. Four trials ${ }^{6-9}$ indicated that levels of IL-6 at 24 hours after CPB were significantly greater in the placebo group than in the methylprednisolone group. However, Keski Nisula et al., ${ }^{11}$ showed that no significant differences were found in the IL-6. Three trials 6-8 reported levels of IL-8. Yilmaz et al., ${ }^{6}$ showed that levels of IL-8 at 24 hours after CPB were no statistically significant difference between the methylprednisolone group and the placebo group. Ayda et al., ${ }^{7}$ showed levels of IL-8 $(p<0.05)$ at 24 hours after CPB were significantly greater in the placebo group than in the methylprednisolone group. Jale Bengi et al., ${ }^{8}$ showed that levels of IL-8 $(p<0.05)$ at 24 hours after CPB were significantly greater in the placebo group than in the methylprednisolone group. Four trials 8-11 reported levels of IL-10. Jale Bengi et al. ${ }^{8}(\mathrm{p}<0.05)$ and Vladimir et al. ${ }^{10}(p<0.05)$ showed that levels of IL-10 were significantly greater in the methylprednisolone group than in the placebo group, but Demir et al., ${ }^{9}$ and Keski-Nisula et al., ${ }^{11}$ showed that there was no statistically significant difference between the methylprednisolone group and the placebo group. According to the overall analysis, methylprednisolone can reduce TNF- $\alpha$, IL- 6 , and IL-8 release. However, we did not know whether methylprednisolone can reduce IL- $1 \beta$ release or increase IL10 release.

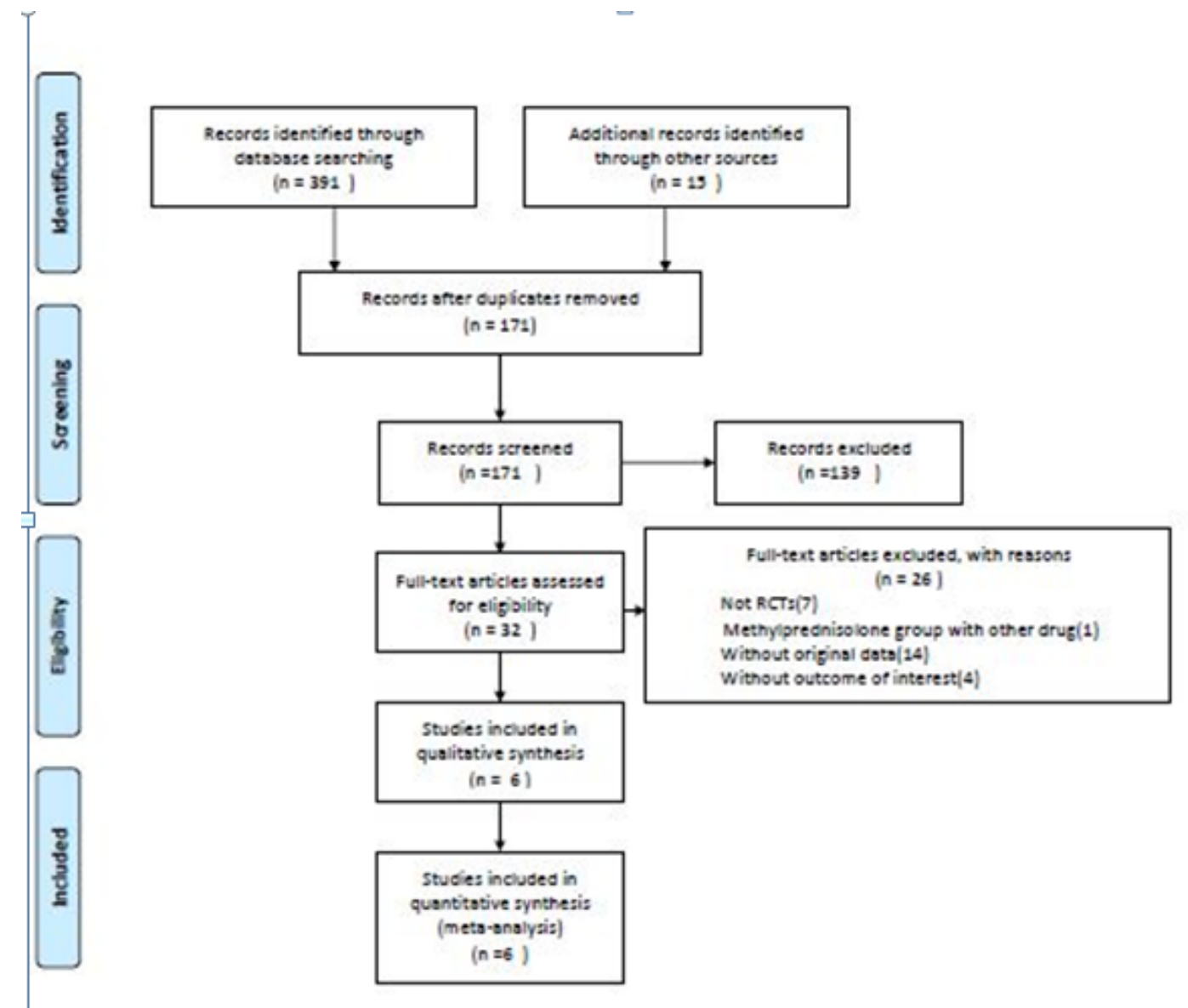

Figure I Flow diagram of included studies. 
Table I Patient characteristics and Jadad score

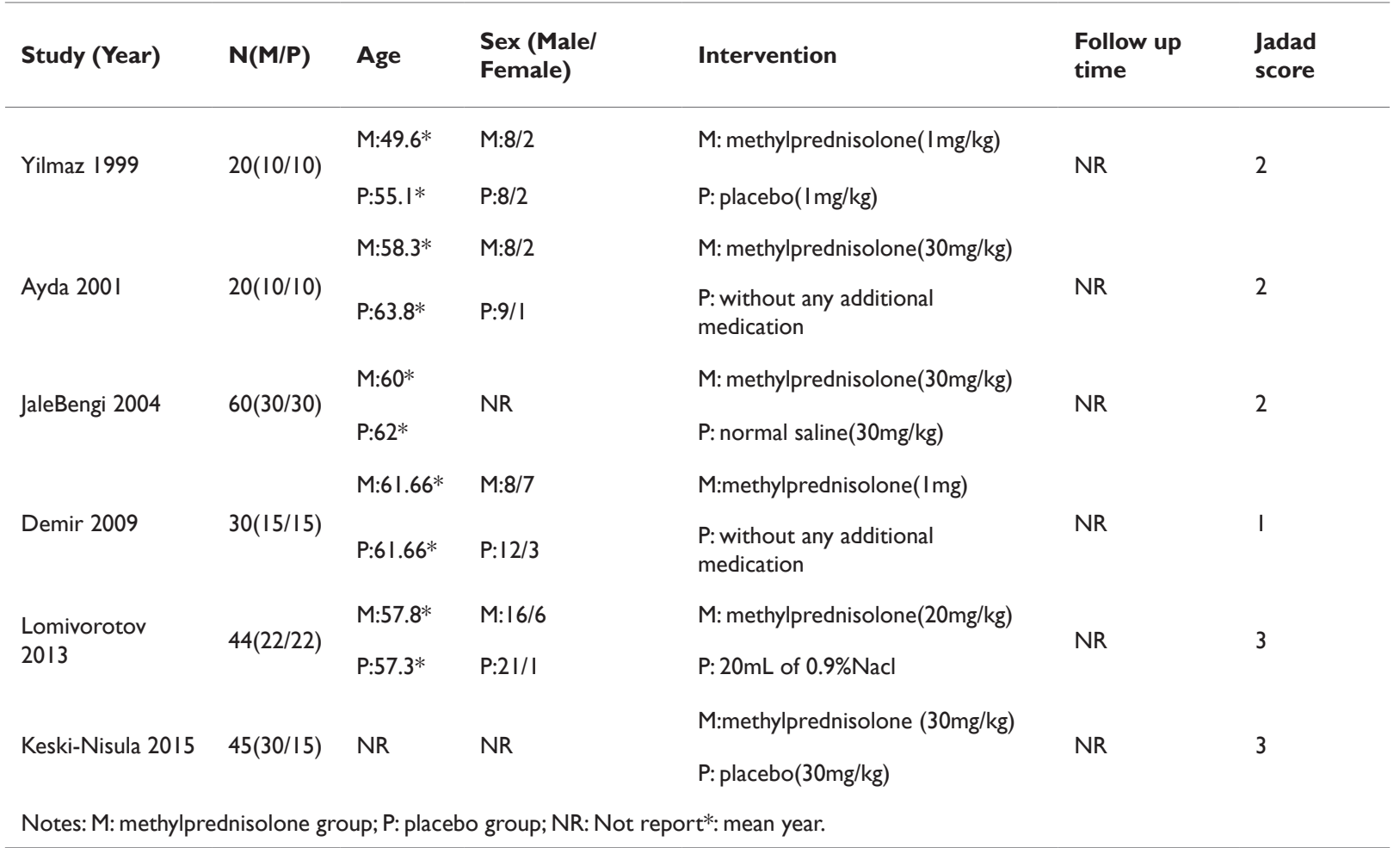

Table 2 The risk of bias of included studies

\begin{tabular}{|c|c|c|c|c|c|c|}
\hline Study(Year) & $\begin{array}{l}\text { Selection } \\
\text { Bias }\end{array}$ & Performance Bias & $\begin{array}{l}\text { Detection } \\
\text { Bias }\end{array}$ & Attrition Bias & Reporting Bias & Other Bias \\
\hline Yilmaz 1999 & High & Unclear & Unclear & Low & Unclear & Unclear \\
\hline Ayda 200I & Unclear & Unclear & Unclear & Low & Unclear & Unclear \\
\hline JaleBengi 2004 & Unclear & Unclear & Unclear & Low & Unclear & Unclear \\
\hline Demir 2009 & Unclear & Unclear & Unclear & Low & Unclear & Unclear \\
\hline Lomivorotov 2013 & Unclear & Unclear & Unclear & Low & Low & Low \\
\hline Keski-Nisula 2015 & Low & Low & Unclear & Low & Low & Low \\
\hline
\end{tabular}

Notes: High: high risk of bias; Low: low risk of bias; Unclear: unclear risk of bias

\section{Discussion}

This systematic review showed that methylprednisolone can restrain TNF- $\alpha$, IL-6, and IL-8 release. Methylprednisolone therapy may reduce the inflammatory response during the CPB. A larger RCT showed that administration of perioperative methylprednisolone in patients, who were at high risk of morbidity and mortality undergoing cardiac surgery with the use of cardiopulmonary bypass, did not reduce the risk of death, or the composite risk of death, myocardial injury, stroke, renal failure, and respiratory failure at 30 days. However, perioperative methylprednisolone in the treatment of patients at high risk of morbidity and mortality undergoing cardiac surgery with the use of cardiopulmonary bypass significant increased the risk of myocardial injury. ${ }^{12}$ In addition, Later et al., ${ }^{13}$ antifibrinolytics had effects on inflammatory response, so the results of the trial were lack of reliability. There are some limitations in this systematic review, hence the reason why this systematic review could only do descriptive analysis. Most studies which were included this systematic review had poor methodological quality. These small-scale studies are not conclusive because of small sample size. Despite, another limitation was caused by lack of gray literature. This systematic review only did descriptive analysis.

\section{Conclusion}

In summary, the current limited evidence shows that methylprednisolone can reduce the inflammatory response during the CPB to a certain degree. Randomized controlled trials which are welldesign and large sample size are required to confirm this conclusion.

\section{Acknowledgements}

None. 


\section{Conflict of interest}

The author declares no conflict of interest.

\section{References}

1. Graham EM, Atz AM, McHugh KE, et al. Preoperative steroid treatment does not improve markers of inflammation after cardiac surgery in neonates:Results from a randomized trial. J Thorac Cardiovasc Surg. 2014;147(3):902-908.

2. Murphy GS, Whitlock RP, Gutsche JT, et al. Steroids for adult cardiac surgery with cardiopulmonary bypass: Update on dose and key randomized trials. J Cardiothorac Vasc Anesth. 2013;27(5):1053-1059.

3. Anić D, Gašparović H, Ivančan V, et al. Effects of corticosteroids on inflammatory response following cardiopulmonary bypass. Croat Med J. 2004;45(2):158-161.

4. Keski-Nisula J, Pesonen E, Olkkola KT, et al. Methylprednisolone in neonatal cardiac surgery: reduced inflammation without improved clinical outcome. Ann Thorac Surg. 2013;95(6):2126-2132.

5. Higgins JP, Altman DG. Chapter 8: Assessing risk of bias in included studies. In: Higgins, Sally Green, editors. Cochrane handbook for systematic reviews of interventions Version 5.1.0. The Cochrane Collaboration. USA: John Wiley \& Sons Ltd; 2011.

6. Yilmaz M, Ener S, Akalin H, et al. Effect of low-dose methyl prednisolone on serum cytokine levels following extracorporeal circulation. Perfusion. 1999;14(3):201-206.
7. Türköz A1, Ciğli A, But K, et al. The effects of aprotinin and steroids on generation of cytokines during coronary artery surgery. $J$ Cardiothorac Vasc Anesth. 2001;15(5):603-610.

8. Celik JB, Gormus N, Okesli S, et al. Methylprednisolone prevents inflammatory reaction occurring during cardiopulmonary bypass:Effects on TNF- $\alpha$, IL-6, IL-8, IL-10. Perfusion. 2004;19(3):185-191.

9. Demir T, Demir H, Tansel T, et al. Influence of methylprednisolone on levels of neuron-specific enolase in cardiac surgery: a corticosteroid derivative to decrease possible neuronal damage. J Card Surg. 2009;24(4):397-403.

10. Lomivorotov VV, Efremov SM, Kalinichenko AP, et al. Methylprednisolone use is associated with endothelial cell activation following cardiac surgery. Heart Lung Circ. 2013;22(1):25-30.

11. Keski-Nisula J, Suominen PK, Olkkola KT, et al. Effect of timing and route of methylprednisolone administration during pediatric cardiac surgical procedures. Ann Thorac Surg. 2015;99(1):180-185.

12. Whitlock RP, Devereaux PJ, Teoh KH, et al. Methylprednisolone in patients undergoing cardiopulmonary bypass (SIRS): A randomised, double-blind, placebo-controlled trial. The Lancet. 2015;386(10000):1243-1253.

13. Later AF, Sitniakowsky LS, van Hilten JA, et al. Antifibrinolytics attenuate inflammatory gene expression after cardiac surgery. $J$ Thorac Cardiovasc Surg. 2013;145:1611-1616. 